\title{
FINITE ELEMENT ANALYSIS OF THERMAL STRESSES IN A PAD-DISC BRAKE SYSTEM (A REVIEW)
}

\author{
Adam ADAMOWICZ*, Piotr GRZES*
}

Faculty of Mechanical Engineering, Department of Mechanics and Applied Computer Science, Bialystok University of Technology ul. Wiejska Street 45C, 15-351 Bialystok, Poland

a.adamowicz@pb.edu.pl, p.grzes@pb.edu.pl

\begin{abstract}
Rapid temperature change in components of the sliding systems induces thermal stresses due to thermal expansion. This effect is particularly evident in disc brakes working under high thermal loads. This paper deals with the finite element modelling of frictional heating process in disc brakes and clutches to study the temperature and stress distributions during operation.
\end{abstract}

Key words: Pad-disc brake system, Temperature, Thermal stresses, Finite element method

\section{INTRODUCTION}

Large amount of mechanical energy converted into heat during braking leads to temperature increase in friction elements (Fig. 1). This may have a significant influence on the thermal behaviour of the brake system. An increase in the temperature above a permissible value, frequently leads to undesirable effects such as fluctuations of the coefficient of friction, brake fade, thermal cracks, thermal deformations of the disc, degradation of the material of the pad, etc. The review articles on analytical methods to study the thermal problems of friction are given in monographs (Chichinadze, 1967; Chichinadze et al., 1979; Balakin and Sergienko, 1999) and in articles (Yevtushenko et al., 2000; Matysiak and Yevtushenko, 2000). The finite element analyses of the transient temperature fields in disc brakes are given in articles by Yevtushenko and Grzes $(2010,2014)$. The authors reported the techniques employed to develop the FE models of the paddisc brake systems and clutches aiming to examine the axisymmetric and three-dimensional temperature distributions during operation. In this paper numerical studies concerning the thermal stresses in disc brakes and clutches are given and the main factors affecting the stress distributions are discussed.

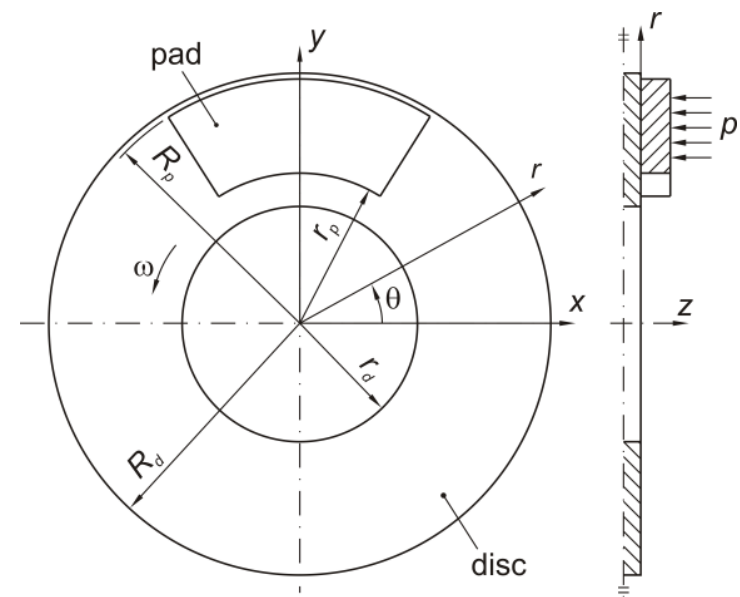

Fig. 1. A schematic diagram of a pad-disc brake system

\section{THERMAL STRESSES IN DISC BRAKES}

The axisymmetric transient temperature and the quasi-static thermal stress distributions in the discs of the wet clutch were determined numerically in article by Zagrodzki (1985). Two different types of the discs were analysed: the first was made entirely of steel, whereas the second was additionally coated with the friction material. At the first step of the study, the temperature fields in both types of the discs (arranged alternately) were calculated using the finite difference method (FDM). Next the thermal stresses using the finite element method (FEM) were determined only for the disc made entirely of steel, which stemmed from its frequent failure during the operation process. The contact pressure on the friction surfaces was constant and uniformly distributed. The convective heat transfer conditions according to Newton's law of cooling in the axisymmetric model were taken into account. As stated it did not have a significant effect on the volume temperature, but might have had affected the change in the local surface temperature and hence the stress. In the area of frictional contact, the temperature equality condition was used and it was assumed that the sum of the heat flux densities is equal to the power density of friction forces. The calculation of the temperature and stresses were uncoupled. Based on the carried out analysis, it was found that the determined maximum equivalent HuberMises stresses did not exceed the yield strength of the tested material. The obtained results were insufficient to predict the damage of the clutch discs. Due to the axial symmetry, the circumferential stress component was at the same time the principal stress component. The directions of the normal stress tensor components, almost coincided with the directions of principal stresses, therefore normal stress components were analysed the as the principal stresses. The order of the magnitude of axial stress was about 30 times lower than the circumferential and the radial stress. Near the friction surface the stresses (circumferential and radial) were compressive. Inside the disc the sign changed to the opposite. During the of the operation of the clutch, the temperature was proportional to the radial coordinate thus the radial and the circumferential stresses also varied in radial direction. The concluding remarks indicated that the essential impact on the value of the circumferential stress component has the radial tem- 
perature gradient resulting from the difference in the relative sliding velocity of the discs. The authors presented this relationship in the diagram. The sign of circumferential stress in the presence of only the component of the axial temperature gradient were shown, then only the radial component and eventually the combined effect of both components were presented. As a result of superposition of the two components of the temperature gradient, the circumferential component of the stress was the highest on the outer surface (compression) and on the inner surface of the disc (tension). After the standstill, a separation between the signs of circumferential component occurred: a positive (inner side) and a negative (outer side). The values of the stress when disconnected discs were not as high as during the engagement of the clutch, however, lasted for a relatively long time. It was pointed out that the effect of the radial component of the temperature gradient on the values and the distribution of the stresses is crucial. It should also be noted, however, that the operating characteristics of the clutch varies from the corresponding characteristics of the disc brake. Thereby the stress distribution and the rate of its change may also differ. The equivalent Huber-Mises stress did not exceeded the yield strength of typical materials used for the clutch discs.

Assuming that the distribution of the contact pressure on the working surfaces of the discs of a wet clutch is uniform and independent of the thermal state of the considered system, the equivalent stress values do not exceed the yield strength under normal operating conditions according to Zagrodzki (1985). An attempt to qualify these assumptions have been made in article (Zagrodzki, 1990). A multidisc clutch consisting of the nine deformable discs made entirely of steel and coated with friction material were analysed. The calculated axisymmetric pressure distributions revealed its decrease with the increase in the radial distance, at the beginning of the operation of the clutch. Further two local extremes were formed, which stemmed from the boundary conditions due to the non-symmetrical geometry of the clutch. In the middle of the clutch system, the discs were heated more intensely than those at the edges (the heating took place only on the one side of the disc). It has also been observed that the discs were subjected to displacements and rotation. The detailed observation revealed that the discs were also folded. This effect has led to the uneven pressure distribution and the formation of the local heating contact areas. The equivalent Huber-Mises stress reached $380 \mathrm{MPa}$. Double reduction in Young's modulus of the friction material allowed to reduce the stress also double. Wear of the material was neglected, which, as stated could weaken the thermoelastic instability phenomenon.

The thermoelastic instability for the pad-disc brake system during a single braking at constant speed was studied by Choi and Lee (2003). The axisymmetric transient temperature fields of the pad and the disc were analysed. An influence of the angular speed and the load acting as the hydraulic pressure on a real contact area and the temperature of the friction elements were examined. The changes in the normal components of the stress tensor were analysed during braking process. Both the compressive and the tensile circumferential stresses increased during braking until the end of the process and it was the highest stress tensor component. Slightly lower values were obtained for the radial component, which after some time remained on the same level.

The coupled thermomechanical analysis for the pad-disc brake system during several applications at linearly decreasing speed was carried out in article by Choi and Lee (2004). The influence of the properties of materials (specific heat, thermal expansion coefficient, thermal conductivity, Young's modulus) on the temperature field and the real contact area was studied. Based on calculations it was concluded that the maximum stress was reached for the circumferential component, and its value was dependent upon the number of brake applications.

The uncoupled thermomechanical analysis for the pad-disc brake system of a rolling stock (TGV) to indicate the factor affecting the disc failure was carried out in article (Dufrenoy and Weichert, 2003). The five major types of thermal gradients generated in the brake disc and their impact on the initiation and growth of the cracks were presented. It was established that the most critical in initiating of the cracks was the distribution of the hot spots in the circumference of the rubbing path, so called buckling mode of the disc. The temperature and the stresses due to the mechanical and the thermal loads were calculated. At the first stage an effect of the mechanical load, resulting from the pressure and the friction force on the stresses in the pad-disc system based on the threedimensional contact model was studied. It was found that the maximum equivalent stress in the pad was several times lower than the limit values and can be neglected in heat conduction problems of friction. Furthermore it was also observed that the obtained stress distribution reflected only the initial state of the braking process. In the next step, the uncoupled thermomechanical analysis was performed. It was assumed that the distribution of the hot spots in the circumference is regular and independent of time. The three-dimensional model of the brake disc was developed. Due to the axial symmetry of the geometry of the disc and a regular distribution of the six hot spots in the circumference of the rubbing path, the calculation area was restricted to 1/12 segment of the disc. First the number of hot spots and the distribution of contact pressure was calculated. Next the parabolic type distribution of the heat flux density of a single spot was applied. The circumferential stress and the plastic deformation using the elastic-plastic model were determined. The outcomes of the FE modelling agreed well with the results of the thermographic measurements. Next, the residual stresses were determined experimentally using the strain gage hole drilling method. An emphasis was placed on the temperature gradients caused by local heating of the disc. The hysteresis loops for multiple braking process were shown. The possibilities of the application of the well-known hypotheses of fatigue in the disc brakes were discussed. The accuracy of assumptions of parabolic distribution of the heat flux densities of regular shapes of hot spots was verified. Applying the temperature boundary condition on the friction surfaces of the disc obtained from thermography measurements, the temperature fields in the disc were calculated numerically.

The thermomechanical macrostructural model of a disc brake of a high-speed rail (TGV) was developed in (Dufrenoy, 2004). The calculations were performed based on the algorithm incorporating varying during braking process the topography of the friction surfaces of the pads (three-dimensional model) and the disc (two or three-dimensional model), wear of the pad, temperaturedependent physical and mechanical properties of materials of the pad and the disc. The uncoupled iterative algorithm was used, according to which, the heat flux partition was determined based on the pressure distribution under static load and taking into account the thermal resistance. Then wear was determined in order to estimate the change in the volume and the topography of the friction surface of the pad, which in turn had an influence on the pressure distributions of the deformable bodies in the next step of mechanical analysis. The process was repeated in cycles. The 
changes in the transient temperature fields of the pad and the disc in the radial direction were determined. The obtained outcomes were compared with the experimental results carried out using thermography techniques and thermocouples.

The problem of the crack initiation and growth in the rail wheels subjected to cyclic thermal loads due to heating and cooling during braking were examined in article (Rossmanith et al., 2006). A mechanism of fracture based on the extensive analysis of the variability of distributions of stress-temperature during braking involving multiple braking to stop, the subsequent cooling, and acceleration to the initial speed of the disc, starting another cycle was studied. The properties of materials (Young's modulus, thermal expansion coefficient and the yield strength) were dependent on the temperature. The convective heat transfer as well as the thermal radiation were included. The heat conduction was restricted to the wheel - the pads in the total energy balance were neglected. The pressure distribution in the heating area depended on the current thermal state of the disc. In order to analyse the actual conditions (real braking process), the multiple braking application was simulated. The one brake application was considered as a single load cycle. Based on the obtained outcomes, it was concluded that the residual stresses resulting from the exceeding of the yield strength, are tensile stress and their values slightly decrease from the inner to the outer surface. To analyse the mechanism of initiation and growth of cracks authors introduced the simplifying assumptions. The crack was modelled as a semi-circular of radius $a$, which retained its shape in the process of propagation. The second assumption was related to the stress field and the volume of the crack.

Finite element analysis of temperature and stress distributions based on the contact thermomechanical FE model of the pad-disc brake system was carried out in article by Scieszka and Zolnierz (2007a, 2007b). The dependencies of the coefficient of friction on the relative sliding velocity and the temperature for the mine winder disc brake using infrared techniques were determined. The results of numerical calculations in the form of transient temperature fields were verified experimentally based on the infrared measurements. The phenomenon of thermoelastic instability was analysed. A braking process was simulated and the formation of hot spots on the working surface of the disc was visualized. The concept of critical speed of the initiation of thermoelastic instability phenomenon was introduced. An attempted was made to establish a method for determining the critical speed based on the developed three-dimensional FE thermomechanical contact model. At the first stage the authors proposed the criterion of $10 \%$ of the temperature difference on the opposite sides of the disc to the initiation of the phenomenon of thermoelastic instability according to (Choi and Lee 2004) and applied to the developed numerical model of the disc brake. Based on the obtained results the dependencies of the critical speed on the thermophysical properties material of the disc and its thickness were shown. It was observed that the greatest impact on the critical speed had the coefficient of thermal expansion of the material of the disc. The decrease in the coefficient of thermal expansion as well as the Young's modulus significantly expanded the range of the critical speed of the initiation of TEI. Also, increasing the thickness of the disc led to an increase in the critical speed. It was observed that despite the initiation of the thermoelastic instability, the brake operation was not disturbed. Therefore a new criterion related to the structural restrictions, namely the size of the gap between the disc and pad was formulated (a criterion for permissible lateral run-out). The critical speed above which the axial displacement exceeded the acceptable range was determined. In the next stage, the effect of the division of the disc on the stress was tested. For this purpose a comparative analysis of equivalent stress of sectional and solid disc during emergency braking was carried out. The distributions of the equivalent stress in the specific braking time were shown. The calculations revealed that the solid disc is subjected to the presence of the equivalent stress exceeding the yield strength of the tested steel type. Whereas this problem did not occur in the sectional disc.

The contact thermomechanical three-dimensional finite element model to study the transient temperature and the stress fields in the pad-disc brake system was developed in article by Gao et al. (2007). The temperature and the stress changes at selected radial positions on the working surface of the disc were determined. A clear relationship between the temperature evolution and the change in equivalent stress was observed. The highest values of both of these quantities were obtained in approximately half of the braking time. The maximum normal stress occurred for circumferential component (compression). Slightly lower values were reached for a radial stress component. The sign of the radial and circumferential stress in the area near the outer cylindrical surface tended to change at the end of braking. This effect was particularly marked in the case of radial stress.

The coupled thermomechanical problem for a multi-disc dry clutch made of a composite material was carried out in (Zhao et al., 2008). The transient temperature and the stress tensor components changes for a single clutch engagement and the disengagement using an axisymmetric contact FE model were calculated. The results were obtained for the operation of the clutch and after the disconnection of the discs. The highest stress values were reached for the circumferential component (compressive stress) in approximately half of the operation time of the clutch.

The identification of the nature and cause of damage of the cast-iron discs of a truck subjected to high thermal loads using the technique of macro-fractography, optical and scanning electron microscopes in article (Bagnoli et al., 2009). With a view to eliminating one of the cause of cracking originated from defects in material of the studied material (cast iron), based on the measurements, the chemical composition, structure and hardness were verified. It was found that the propagation of radial macro-cracks in the heating area of semi-elliptic shape extends from the surface into the disc as a result of thermal fatigue mechanism. Then the changes in temperature distributions and equivalent Huber-Mises stress in the disc were calculated numerically using FEM. The calculations confirmed the presence of high temperature gradients in the area of the friction surface resulting in a local increase in the Huber-Mises stress.

The transient uncoupled temperature field and the thermal stresses were analysed in the article (Collignon et al., 2013). An attempt was made to determine the type and size of the thermo-mechanical load of components of the friction pair causing damage of the discs in the form of radial macro-cracks. On a developed for this purpose bench, a series of tests of the brake system according to an algorithm established on the assumption that the mechanical energy is entirely transformed into heat during braking. A new unused disc was loaded recording changes in temperature field by using an infrared camera and thermocouples. The numerical calculations were carried out using ANSYS FE numerical package. In the model comprising 1/20 of the ventilated disc, the temperature-dependent thermophysical properties of the material were taken into account. Moreover, on the surfaces free from heating the heat exchange through thermal radiation and 
convective cooling at the different values of heat transfer coefficient depending on the position took place. Elastic-plastic model was adopted for the material (cast iron) introducing beyond the established within the software the same behaviour in compression and tension, two different criteria: Huber-Mises (compression) and Rankine (tension). Temperature fields in different moments during the analysed braking process at the chosen time step were analysed. On the basis of the obtained temperature distributions thermal stresses were calculated. Slight differences in temperature changes calculated numerically and recorded during the experimental tests were justified by possible different cooling conditions of the brake system.

The comparative analysis of stress distribution in a ventilated disc with uniform and non-uniform distribution of the contact pressure was performed in article (Kim et al., 2008). The pressure distribution was determined numerically based on the contact three-dimensional computational model incorporating exclusively mechanical interactions. Based on calculations it was shown that the pressure distribution was relatively uniform, however the highest value of the pressure in the radial direction was obtained in the centre of the rubbing path. With the distance to both the outer and the inner cylindrical surface of the disc, the pressure decreased. The resulting pressure distribution was then treated as a boundary condition in the thermal analysis. A simulation of a single braking process at constant deceleration was carried out. Analysis of stress fields in the case of non-uniform pressure distribution revealed the highest values of equivalent Huber-Mises stress on the friction surface near the centre of the rubbing path. If the pressure distribution was uniform, the maximum stress values were slightly higher (about 3\%), and occurred near the inner circular edge of the disc on the friction surface.

A strength of disc brakes with a circular holes (joints) made of a composite carbon/carbon quasi-isotropic material were studied in article (Lim et al., 2008). The specimens with different angles regarding the reference axis were extracted from a real disc brake and their properties were derived from the tension, compression and shear tests. The failure criterion was formulated on the basis of the maximum stress theory. The failure loads were measured and applied to the three-dimensional model of a specimen with a hole. The numerical calculations were performed using MSC Marc software.

A relatively low number of braking cycles with a significant deceleration may cause a strain leading to plastic stress. An attempt to determine the number of cycles to failure based on the CoffinManson criterion used under low cycle fatigue loads was taken in article (Mackin et al, 2002). In the first stage, the forces acting on the elements of the friction pair during braking were determined. Then the braking conditions (braking time, the initial velocity, the number of brake applications) that may lead to the development of the thermal cracks were set. The numerical calculations to obtain the temperature distributions were carried out using FEM. Based on the calculated radial, circumferential and equivalent HuberMises stress as well as the Coffin-Manson formula the number of cycles to the appearance of damage of the brake disc was predicted. The values obtained from the calculations were higher about $11 \%$ compared to the data evaluated from experimental studies. Moreover, the maximum equivalent stresses exceeded the yield strength of the tested material near the hub. According to (Mackin et al., 2002) during braking compressive stresses prevail, while during cooling the tensile residual circumferential stress are dominant. The repeating cycle of the successive states of stress comprises a mechanism of a fatigue leading to a failure.
Based on observation with a scanning electron microscope three types of structures of the material were identified defining the successive steps of cracks growth in the disc brake in article (Sakamoto and Hirakawa, 2005). The impact of the chemical composition to improve the properties of the materials used for the brake discs was discussed. This was followed by FE numerical simulation of a braking process in the axisymmetric arrangement. The results were compared with the strain measurements. Based on the calculated stress distribution, the stress intensity factors were determined. In addition, the stress intensity factors were determined from measurements carried out on CT (compact tension) specimens. The heating cycle goes from the compressive stress at the time of reaching the maximum temperature then the residual tensile stress at the decrease in temperature to the room temperature. Next, a discussion of possibilities to improve the breaking strength by changing the chemical composition of the material was carried out. Pointed out the possibility of using different methods, such as Coffin, Manson, Northcott, Blauela and other thermal fatigue tests, however, none of the methods, as stated do not provide for changes in many factors occurring during braking and require simplification.

The stress fields in a ventilated and a solid type of the brake discs using the developed fully three-dimensional FE models were studied in article by Yildiz and Duzgun (2010). The analysis was performed taking into account only the mechanical loads without the generation of heat due to friction. Three different types of ventilated and drilled brake discs different in the arrangement and shape of the holes and a solid disc with a uniform distribution of clamping force were examined. Then the modification of the distribution of force on the pad, in order to determine the most favourable from the point of view of minimizing the equivalent stress on the brake disc was proposed. It was observed that the higher stresses were induced in a ventilated brake disc compared to the solid disc.

\section{CONCLUSIONS}

The paper presents the finite elements analyses of the thermal stresses in disc brakes and clutches. The calculations were performed using the axisymmetric and fully three-dimensional computational models. The following conclusions on the basis of the analysed studies have been drawn:

- the contact pressure distribution (either assumed as constant in thermal analysis or calculated in contact thermomechanical analysis) affects the temperature field on the friction surfaces and changes the location of the highest equivalent HuberMises stress;

- the circumferential and radial stress components calculated both using the contact thermomechanical models as well as when considering two bodies separately, are dominant normal stress components and are approximately equal;

- the axial stress component is frequently neglected in the analysis of the results due to its relatively small value;

- in order to minimize the radial and being its result, the circumferential stress component, the discrepancy in the radial distribution of the heat flux density should be minimized;

- the problem of the fatigue failure of the disc brakes is not entirely explored and leaves some open questions due to the difficulty in employing directly the fatigue criterions to the brake discs. 


\section{REFERENCES}

1. Bagnoli F., Dolce F., Bernabei M. (2009), Thermal fatigue cracks of fire fighting vehicles gray iron brake discs, Engineering Failure Analysis, Vol. 16, 152-163.

2. Balakin V.A., Sergienko V.P. (1999) Heat calculations of brakes and friction units, MPRI of NASB, Gomel, (in Russian).

3. Chichinadze A.V. (1967), Calculation and investigation of external friction during braking, Nauka, Moscow, (in Russian).

4. Chichinadze A.V., Braun E.D., Ginsburg A.G., Ignat'eva E.V. (1979), Calculation, test and selection of frictional couples, Nauka, Moscow, (in Russian).

5. Choi J-H., Lee I. (2003), Transient thermoelastic analysis of disk brakes in frictional contact, Journal of Thermal Stresses, Vol. 26, No. 3, 223-244.

6. Choi J-H., Lee I. (2004), Finite element analysis of transient thermoelastic behaviors in disk brakes, Wear, Vol. 257, 47-58.

7. Collignon M., Cristol A-L., Dufrénoy P., Desplanques Y., Balloy D. (2013), Failure of truck brake discs: A coupled numericalexperimental approach to identifying critical thermomechanical loadings, Tribology International, Vol. 59, 114-120.

8. Dufrénoy P. (2004), Two-/three-dimensional hybrid model of the thermomechanical behaviour of disc brakes, Proceedings of the Institution of Mechanical Engineers, Part F: Journal of Rail and Rapid Transit, Vol. 218, No. 1, 17-30.

9. Dufrénoy P., Weichert D. (2003), A thermomechanical model for the analysis of disc brake fracture mechanisms, Journal of Thermal Stresses, Vol. 26, 815-828.

10. Evtushenko O.O., Ivanyk E.H., Horbachova N.V. (2000), Analytic methods for thermal calculation of brakes (review), Materials Science, Vol. 36, No. 6, pp. 857-862.

11. Gao C.H., Huang J.M., Lin X.Z., Tang X.S. (2007), Stress analysis of thermal fatigue fracture of brake disks based on thermomechanical coupling, ASME Journal of Tribology, Vol. 129, No. 3, 536-543.

12. Kim D.-J., Lee Y.-M., Park J.-S., Seok C.-S. (2008), Thermal stress analysis for a disk brake of railway vehicles with consideration of the pressure distribution on a frictional surface, Materials Science and Engineering: A, Vol. 483-484, 456-459.

13. Lim D.-W., Kim T.-H., Choi J.-H., Kweon J.-H., Park H.-S. (2008), A study of the strength of carbon-carbon brake disks for automotive applications, Composite Structures, Vol. 86, No. 1-3, 101-106.

14. Mackin T.J., Noe S.C., Ball K.J., Bedell B.C., Bim-Merle D.P., Bingaman M.C., Bomleny D.M., Chemlir G.J., Clayton D.B., Evans H.A., Gau R., Hart J.L., Karney J.S., Kiple B.P., Kaluga R.C., Kung P., Law A.K., Lim D., Merema R.C., Miller B.M., Miller T.R., Nielson T.J., O'Shea T.M., Olson M.T., Padilla H.A., Penner B.W., Penny C., Peterson R.P., Polidoro V.C., Raghu A., Resor B.R., Robinson B.J., Schambach D., Snyder B.D., Tom E., Tschantz R.R., Walker B.M., Wasielewski K.E., Webb T.R., Wise S.A., Yang R.S., Zimmerman R.S. (2002), Thermal cracking in disc brakes, Engineering Failure Analysis, Vol. 9, 63-76.
15. Matysiak S.J., Yevtushenko A.A. (2001) On heating problems of friction, Journal of Theoretical and Applied Mechanics, Vol. 3, No. 39, 577-588.

16. Rossmanith H.P., Loibnegger F., Huber R. (2006), Thermomechanical fatigue fracture due to repeated braking of railway wheels, Materials Science, Vol. 42, No. 4, 40-48.

17. Sakamoto H., Hirakawa K. (2005), Fracture analysis and materia improvement of brake discs, JSME International Journal Series $A$, Vol. 48, No. 4, 458-464.

18. Scieszka S., Zolnierz M. (2007a), The effect of the mine winder disc brake's design feature on its thermoelastic instability. Part I. Set-up for finite element modelling and numerical model verification, Problems of Machines Operation and Maintenance, Vol. 42, No. 3 , $111-124$.

19. Scieszka S., Zolnierz M. (2007b), The effect of the mine winder disc brake's design feature on its thermoelastic instability. Part II. Finite element simulation, Problems of Machines Operation and Maintenance, Vol. 42, No. 4, 183-193.

20. Yevtushenko A.A., Grzes P. (2010), The FEM-modeling of the frictional heating phenomenon in the pad/disc tribosystem (a review), Numerical Heat Transfer, Part A: Applications, Vol. 58, No. 3, $207-$ 226.

21. Yevtushenko A.A., Grzes P. (2014), FEM-modeling of frictional heating during braking, Encyclopedia of Thermal Stresses, Vol. 4 Springer Science+Business Media Dordrecht, DOI 10.1007/978-94007-2739-7.

22. Yildiz Y., Duzgun M. (2010), Stress analysis of ventilated brake discs using the finite element method, International Journal of Automotive Technology, Vol. 11, No. 1, 133-138.

23. Zagrodzki P. (1985), Numerical analysis of temperature fields and thermal stresses in the friction discs of a multidisc wet clutch, Wear, Vol. 101, 255-271.

24. Zagrodzki P. (1990), Analysis of thermomechanical phenomena in multidisc clutches and brakes, Wear, Vol. 140, No. 2, 291-308.

25. Zhao S., Hilmas G.E., Dharani L.R. (2008), Behavior of a composite multidisk clutch subjected to mechanical and frictionally excited thermal load, Wear, Vol. 264, 1059-1068.

The work has been accomplished under the research project No. 2011/01/B/ST8/07446 financed by the National Science Centre in Poland (research project). 